\title{
Applications of Stochastic Calculus to Finance
}

\author{
Scott Stelljes \\ University of North Florida
}

Follow this and additional works at: https://digitalcommons.unf.edu/etd

Part of the Mathematics Commons

\section{Suggested Citation}

Stelljes, Scott, "Applications of Stochastic Calculus to Finance" (2004). UNF Graduate Theses and

Dissertations. 267.

https://digitalcommons.unf.edu/etd/267

This Master's Thesis is brought to you for free and open access by the Student Scholarship at UNF Digital Commons. It has been accepted for inclusion in UNF Graduate Theses and Dissertations by an authorized administrator of UNF Digital Commons. For more information, please contact Digital Projects.

(C) 2004 All Rights Reserved

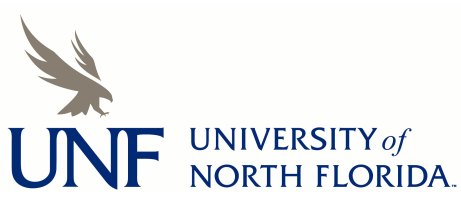




\section{Applications of Stochastic Calculus to Finance}

by

Scott Stelljes

A thesis submitted to the Department of Mathematical Sciences in partial fulfillment of the requirements for the Degree of

Master of Science in Mathematics

University of North Florida

College of Arts and Sciences

November, 2004 


\section{Certificate of Approval}

The thesis of Scott Timothy Stelljes is approved:

(Date)

Signature Deleted

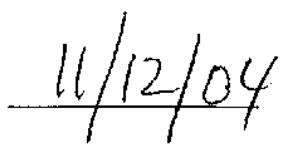

Signature Deleted

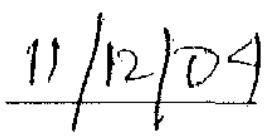

Signature Deleted

$11 / 15 / 04$

Signature Deleted

$11 / 15 / 04$

Committee Chairperson

Accepted for the Department:

Signature Deleted

Chairperson

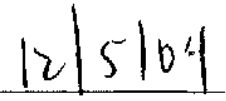

Accepted for the College:

Signature Deleted

Dean

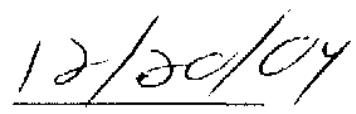

Accepted for the University:

Signature Deleted

Dean of Graduate Studies

1/2tog 


\section{Dedication}

This thesis is dedicated to my wife, Elizabeth Stelljes and my daughter, Amber Stelljes. Without their constant support and patience, I would not have been able to finish this thesis.

\section{Acknowledgements}

I would like to acknowledge my thesis advisor, Dr. Denis Bell and the other faculty of the University of North Florida who assisted with the research of this thesis. Specifically, I would like to acknowledge Dr. Daniel Dreibelbis for developing the numerical method we used to fit data to the fractional model and Dr. Pali Sen for her advice on fitting data to the geometric Brownian motion model. 


\section{Table of Contents}

1. Introduction

2. Mathematical Background

$\begin{array}{llr}2.1 & \text { The Ito Integral } & 4 \\ 2.2 & \text { Ito's Formula } & 11 \\ 2.3 & \text { Girsanov's Theorem } & 20 \\ 2.4 & \text { Stratonovich Calculus } & 23 \\ 2.5 & \text { Existence and Uniqueness of Solutions of Stochastic } & 24\end{array}$

3. Financial Models

$\begin{array}{lll}3.1 & \text { Assumptions } & 26\end{array}$

3.2 Pricing a European Call Option - Risk Neutral
Pricing Method

3.3 Risk Neutral Pricing with Geometric Brownian

3.4 Pricing a European Call Option - Partial Differential

4. Alternative Stock Models

4.1 The Cox and Ross Model 42

4.2 Stock Model Using a Stratonovich Differential 45

5. Fitting Data to Stock Models 50

6. Conclusions $\quad 55$

$\begin{array}{ll}\text { References } & 57\end{array}$

$\begin{array}{ll}\text { Vita } & 58\end{array}$

\section{List of Tables}

$\begin{array}{ll}\text { Table 3.2.1 } & 30\end{array}$

$\begin{array}{ll}\text { Table 3.2.2 } & 30\end{array}$

Table 5.1 Sample of Standard and Poor 100 Stocks 54 


\begin{abstract}
Stochastic Calculus has been applied to the problem of pricing financial derivatives since 1973 when Black and Scholes published their famous paper "The Pricing of Options and Corporate Liabilities" in the Journal of Political Economy. The purpose of this thesis is to show the mathematical principles underlying the methods applied to finance and to present a new model of the stock price process.
\end{abstract}

As part of this paper, we present proofs of Ito's Formula and Girsanov's Theorem which are frequently used in financial applications. We demonstrate the application of these thcorems to calculating the fair price of a European call option. There are two methods that result in the same price: the risk neutral valuation and the Black-Scholes partial differential equation.

A new model of the stock price process is presented in Section 4. This model was inspired by the model of Cox and Ross published in 1976. We develop the model such that a martingale measure will exist for the present value of the stock price. We fit data to the traditional geometric Brownian motion model and the new model and compare the resulting prices. The data fit some stocks well, but in some cases the new model provided a better fit. The price of a European call is calculated for both models for several different stocks. 


\section{Introduction}

The purpose of this thesis is to present rigorous mathematical justification for models used by financial professionals to price financial derivatives and to present an alternative model of the stock price process to be used in pricing derivatives. Derivatives are securities with payoffs that depend on the value of some underlying asset. Examples of these include European and American calls and puts, forwards, and futures. The mathematical models used to price these securities have become quite sophisticated since the 1970 s and make practical use of advanced mathematical theory. This theory includes stochastic calculus and the theory of partial differential equations. This thesis will present the mathematical background for these pricing models with comprehensive proofs, show the development of the models, and test the reliability of the models with historical data. Most of this thesis is an exposition of well established methodologies used in finance with the exception of the model presented in section 4.2 which is the work of the author and Dr. Denis Bell. Please see the references for suggested reading.

The thesis is divided into sections as follows:

\section{Section 2}

The mathematical framework for derivative pricing models is the theory of stochastic calculus. In order to develop this theory rigorously, we will present the definition of the Ito integral and the proof of its existence for a large class of integrands, $\mathcal{L}^{2}$. We will then prove some fundamental theorems of stochastic calculus including the stochastic product 
rule, Ito's Formula, Girsanov's Theorem, and the conditions for existence and uniqueness of solutions of stochastic differential equations.

\section{Section 3}

Financial models for pricing derivatives will be developed from the mathematical theory. To demonstrate the theoretical framework with a simple example, the Black-Scholes formula for pricing European call options will be developed. The price of a European call will be developed using two methods:

- Risk Neutral pricing method, and by

- Solving a Partial Differential Equation with boundary conditions

The same model of stock prices underlies both of these methodologies and they are shown to produce the same result.

\section{Section 4}

Two alternative stock models will be presented along with the price of a European call based on these models. One of these models is based on a paper by Cox and Ross [6] who solved for the price of a European call in an infinite series. Their methodology was very advanced and beyond the scope of this thesis. The model devised by Bell and Stelljes is much easier to implement. 
$\underline{\text { Section } 5}$

Data will be fitted to the stock models presented in sections 3 and 4 . The estimated parameters will be used to price a European call option.

\section{Section 6}

Conclusions. 


\section{Mathematical Background}

\subsection{The Ito Integral}

In this section, we will present the definition of the Ito integral and prove its existence for a class of integrands. The theory presented in this section is taken from Friedman [1] with the exception of the proof of Girsanov's Theorem which is the work of Dr. Denis Bell.

\section{Definition 1: Brownian Motion}

Let $(\Omega, \mathcal{F}, P)$ be a probability space with sample space $\Omega$, sigma-algebra $\mathcal{F}$, and probability measure $P$. A Brownian motion is a stochastic process $w:[0, \infty) \times \Omega \rightarrow \mathbb{R}$ with the following properties:

(1) $w(0)=0$.

(2) $E[w(t)]=0$.

(3) $w(t)$ has stationary increments: $w(t+s)-w(s) \sim w(t)$.

(4) $w(t)$ has independent increments: for $0 \leq t_{1}<t_{2} \leq t_{3}<t_{4}, w\left(t_{4}\right)-w\left(t_{3}\right)$ and $w\left(t_{2}\right)-w\left(t_{1}\right)$ are independent.

(5) The sample path: $t \rightarrow w(t)$ is continuous almost surely.

The above definition of Brownian motion results in the following additional properties:

(1) $w(t) \sim N(0, c t)$ where $c$ is a positive constant. We assume throughout that $c=1$. 
(2) Sample paths are nowhere differentiable almost surely.

(3) Brownian motion has unbounded variation almost surely on any interval.

In particular, the stochastic integral $\int_{i_{1}}^{t_{2}} f(s) d w(s)$ cannot be defined as a

Riemann-Stieltjes integral.

\section{Definition 2: The Class of Integrands for the Ito Integral}

The class of integrands for which the Ito integral will be defined is denoted $\mathcal{L}^{2}[0, T]$. It is the set of functions, $f$ such that

(1) $f$ is jointly measurable on $[0, T] \times \Omega$, i.e. for any Borel set $B \subset \mathbb{R}$, $f^{-1}(B) \in \sigma([0, T] \times \mathcal{F})$

(2) $f$ is adapted (non-anticipating), i.e. $f(t, w)$ depends only on $\{w(s): s \leq t\}$.

(3) $E\left[\int_{0}^{T} f^{2}(s) d s\right]<\infty$.

For any $f$ and $g \in \mathcal{L}^{2}$ we define an inner product $\langle f, g\rangle \equiv E\left[\int_{0}^{T} f(s) g(s) d s\right]$ and a norm $\|f\|=\langle f, f\rangle^{1 / 2}=\left(E\left[\int_{0}^{f} f^{2}(s) d s\right]\right)^{1 / 2}$, It can be shown that $\mathcal{L}^{2}$ is complete with respect to this norm. Thus, $\mathcal{L}^{2}[0, T]$ is a Hilbert space.

We now define a subclass $\mathcal{E} \subset \mathcal{L}^{2}[0, T]$ of random step functions that have the following additional property: 
(4) There is a deterministic partition, $0=t_{0}<t_{1}<t_{2}<\ldots<t_{n}=T$ such that

$$
f(t)=f\left(t_{i}\right) \text { for } t_{i}<t \leq t_{i+1} .
$$

We will need to define a filtration:

\section{Definition 3: Filtration}

A filtration, $\mathcal{F}_{t}=\sigma(w(s): s \leq t)$, the $\sigma$-algebra generated by the random variable $\{w(s): 0 \leq s \leq t\}$

\section{Definition of the Ito Integral for Step Functions}

For a function $f=\sum_{i=0}^{n} f\left(t_{i}\right) \chi_{\left(t_{i}, t_{i+1}\right]}$ where $f\left(t_{i}\right) \in \mathcal{E}$, the Ito integral is defined as

$$
\int_{0}^{T} f(s) d w(s)=\sum_{i=0}^{n-1} f\left(t_{i}\right)\left[w\left(t_{i+1}\right)-w\left(t_{i}\right)\right] .
$$

\section{Definition 4: The Space $L^{2}$}

The space $L^{2}$ is defined as $L^{2}=\left\{X: \Omega \rightarrow \mathbb{R}, E\left[X^{2}\right]<\infty\right\}$.

A norm can be defined on $L^{2}$ as follows: $\|X\|_{2}^{2}=E\left[X^{2}\right]$.

The following lemma reIates the norm on $L^{2}$ to the norm on $\mathcal{L}^{2}[0, T]$. 


\section{Lemma 2.1.1}

For $f \in \mathcal{E}$

(i) $E\left[\int_{0}^{T} f d w\right]=0$.

(ii) $E\left[\left(\int_{0}^{T} f d w\right)^{2}\right]=E\left[\int_{0}^{T} f^{2}(t) d t\right]=\|f\|^{2}$.

i.e. $\left\|\int_{0}^{T} f d w\right\|_{2}^{2}=\|f\|^{2}$.

\section{Proof:}

Using properties (2), (3) and (4) in Definition 1, we have

(i) $E\left[\int_{0}^{T} f d w\right]=\sum_{i=0}^{n-1} E\left[f\left(t_{i}\right)\left(w\left(t_{i+1}\right)-w\left(t_{i}\right)\right)\right]$

$$
\begin{aligned}
& =\sum_{i=0}^{n-1} E\left[E\left(f\left(t_{i}\right)\left(w\left(t_{i+1}\right)-w\left(t_{i}\right)\right) \mid \mathcal{F}_{i}\right)\right] \\
& E\left[\int_{0}^{T} f d w\right]=E\left[\sum_{i=0}^{n-1} f\left(t_{i}\right) E\left[\left(w\left(t_{i+1}\right)-w\left(t_{i}\right)\right) \mid \mathcal{F}_{i_{i}}\right]\right] \\
& =\sum_{i-0}^{n-1} E\left[f\left(t_{i}\right) E\left[w\left(t_{i+1}\right)-w\left(t_{i}\right)\right]\right] \\
& =0
\end{aligned}
$$

because $f\left(t_{i}\right)$ is $\mathcal{F}_{i_{i}}$ measurable.

(ii) $E\left[\left(\int_{0}^{T} f d w\right)^{2}\right]=E\left[\sum_{i, j} f\left(t_{i}\right) f\left(t_{j}\right)\left[w\left(t_{i+1}\right)-w\left(t_{i}\right)\right]\left[w\left(t_{j+1}\right)-w\left(t_{j}\right)\right]\right]$

$$
=\sum_{i, j} E\left[f\left(t_{i}\right) f\left(t_{j}\right) \Delta_{i} w \Delta_{j} w\right]
$$


where $\Delta_{i} w \equiv w\left(t_{i+1}\right)-w\left(t_{i}\right)$.

Case $i \neq j$ : We assume without loss of generality that $i<j$. Then,

$$
E\left[f\left(t_{i}\right) f\left(t_{j}\right) \Delta_{i} w \Delta_{j} w\right]=E\left[E\left[f\left(t_{i}\right) f\left(t_{j}\right) \Delta_{i} w \Delta_{j} w \mid \mathcal{F}_{t_{j}}\right]\right]
$$

since $f\left(t_{i}\right), f\left(t_{j}\right)$ and $\Delta_{i} w$ are measurable with respect to $\mathcal{F}_{t_{j}}$. Therefore,

$$
\begin{aligned}
& E\left[f\left(t_{i}\right) f\left(t_{j}\right) \Delta_{i} w \Delta_{j} w\right]=f\left(t_{i}\right) f\left(t_{j}\right) \Delta_{i} w E\left[\Delta_{j} w \mid \mathcal{F}_{t_{j}}\right] \\
& =0 \text { since } E\left[\Delta_{j} w \mid \mathcal{F}_{t_{j}}\right]=0 \text { by the independent increment property of Brownian }
\end{aligned}
$$

motion.

Case $i=j$ :

$$
\begin{aligned}
& E\left[f^{2}\left(t_{i}\right)\left(\Delta_{i} w\right)^{2}\right]=E\left[E\left[f^{2}\left(t_{i}\right)\left(\Delta_{i} w\right)^{2} \mid \mathcal{F}_{t_{i}}\right]\right] \\
& =E\left[f^{2}\left(t_{i}\right) E\left[\left(\Delta_{i} w\right)^{2} \mid \mathcal{F}_{t_{i}}\right]\right] \\
& =E\left[f^{2}\left(t_{i}\right) E\left[\left(w\left(t_{i+1}\right)-w\left(t_{i}\right)\right)^{2}\right]\right] \\
& =E\left[f^{2}\left(t_{i}\right)\right]\left(t_{i+1}-t_{i}\right) \text { also by the properties of Brownian motion. }
\end{aligned}
$$

Combining the two cases,

$E\left[\left(\int_{0}^{T} f d w\right)^{2}\right]=E\left[\sum_{i} f^{2}\left(t_{i}\right)\left(t_{i+1}-t_{i}\right)\right]=E\left[\int_{0}^{T} f^{2}(t) d t\right] \mathbf{\text { . }}$ 


\section{Lemma 2.1.2}

$\mathcal{E}$ is dense in $\mathcal{L}^{2}[0, \mathrm{~T}]$ with respect to \|\| . In other words, given any

$f \in \mathcal{L}^{2}[0, T], \exists\left\{f_{n}\right\} \subset \mathcal{E}$ such that

$\left\|f-f_{n}\right\| \rightarrow 0$ as $\mathrm{n} \rightarrow \infty$.

Proof:

See Friedman [1].

Given Lemma 2.1.1 and Lemma 2.1.2, we can define the Ito integral for any $f \in \mathcal{L}^{2}[0, T]$ as follows. Choose a sequence $\left\{f_{n}\right\} \subset \mathcal{E}$ as in Lemma 2.1.2. Then $\left\{f_{n}\right\}$ is a Cauchy sequence in $\mathcal{L}^{2}[0, T]$. Thus

$$
\begin{aligned}
& E\left[\left(\int_{0}^{T} f_{n} d w-\int_{0}^{T} f_{m} d w\right)^{2}\right]=E\left[\left(\int_{0}^{T}\left(f_{n}-f_{m}\right) d w\right)^{2}\right] \\
& =E\left[\int_{0}^{T}\left(f_{n}-f_{m}\right)^{2}(t) d t\right] \text { by Lemma } 2.1 .1 \\
& =\left\|f_{n}-f_{m}\right\|^{2} \rightarrow 0 \text { as } n, m \rightarrow \infty
\end{aligned}
$$

It follows that $\int_{0}^{T} f_{n} d w$ is a Cauchy sequence in $L^{2}$. Thus $\int_{0}^{T} f_{n} d w$ has a limit in $L^{2}$. The Ito integral is defined as $\lim _{n \rightarrow \infty} \int_{0}^{T} f_{n} d w$. 
The integral is independent of the choice of the approximating sequence, $\left\{f_{n}\right\}$. To show this, let $\left\{g_{n}\right\}$ be another sequence of step functions with $\left\|f-g_{n}\right\| \rightarrow 0$. Then $\left\|f_{n}-g_{n}\right\| \rightarrow 0$ and we have

$E\left[\left(\int_{0}^{T} f_{n} d w-\int_{0}^{T} g_{n} d w\right)^{2}\right]=E\left[\left(\int_{0}^{T}\left(f_{n}-g_{n}\right) d w\right)^{2}\right]$

$=\left\|f_{n}-g_{n}\right\|^{2} \rightarrow 0$.

Thus $\lim _{n \rightarrow \infty} \int_{0}^{T} f_{n} d w=\lim _{n \rightarrow \infty} \int_{0}^{T} g_{n} d w$.

\section{Properties of the Ito Integral}

(1) Linearity: let $a, b \in \mathbb{R}$ and $f, g \in \mathcal{L}^{2}[0, T]$. Then $a f+b g \in \mathcal{L}^{2}$ and

$\int_{0}^{T}(a f+b g) d w=a \int_{0}^{T} f d w+b \int_{0}^{T} g d w$ a.s.

(2) If $0<t_{1}<t_{2}, f \in \mathcal{L}^{2}$ then $\int_{0}^{t_{2}} f d w=\int_{0}^{t_{5}} f d w+\int_{i_{1}}^{t_{2}} f d w$.

(3) If $f \in \mathcal{L}^{2}$ then $E\left[\int_{0}^{T} f d w\right]=0$ and $E\left[\left(\int_{0}^{T} f d w\right)^{2}\right]=E\left[\int_{0}^{T} f^{2}(s) d s\right]$.

(4) For $f \in \mathcal{L}^{2}[0, T]$, define $\xi(s) \equiv \int_{0}^{s} f d w \equiv \int_{0}^{T} f \chi_{[0, s]} d w$. Then there exists a version of the process $\xi$ such that $s \rightarrow \xi(s)$ is continuous almost surely. See Friedman [1] for a proof. 
The Ito integral does not follow the same chain rule as the Riemann-Stieltjes integral. For example, it is not true that $\int_{t_{1}}^{t_{2}} w(s) d w(s)=\frac{1}{2}\left[\left(w\left(t_{2}\right)\right)^{2}-\left(w\left(t_{1}\right)\right)^{2}\right]$. In the next section, we will give the correct version of this formula.

\subsection{Ito's Formula}

Ito's Formula, the chain rule for Ito integrals, is central to the theory of stochastic calculus. In this section, we will present and prove Ito's Formula. This will require several preliminary results, which we now state and prove.

\section{Lemma 2.2.1}

Let $w(t)$ be a Brownian motion and let $\prod_{n}$ be a sequence of partitions of the interval $[a, b]$ with mesh $\left|\Pi_{n}\right| \rightarrow 0$ as $n \rightarrow \infty$. Define $S_{n} \equiv \sum_{j=1}^{m_{n}}\left[w\left(t_{n, j}\right)-w\left(t_{n, j-1}\right)\right]^{2}$. Then $S_{n} \rightarrow b-a$ in the mean.

Proof:

Let $t_{j}=t_{n, j}$ and $m=m_{n}$ for each $n$. Then,

$$
\begin{aligned}
& S_{n}-(b-a)=\sum_{j=1}^{m}\left[\left(w\left(t_{j}\right)-w\left(t_{j-1}\right)\right)^{2}-\left(t_{j}-t_{j-1}\right)\right] \\
& E\left[\left(S_{n}-(b-a)\right)^{2}\right]=E\left[\left\{\sum\left[\left(w\left(t_{j}\right)-w\left(t_{j-1}\right)\right)^{2}-\left(t_{j}-t_{j-1}\right)\right]^{2}\right\}\right]
\end{aligned}
$$


Define $Y_{j}=\frac{w\left(t_{j}\right)-w\left(t_{j-1}\right)}{\sqrt{t_{j}-t_{j-1}}}$

$E\left[\left(S_{n}-(b-a)\right)^{2}\right]=E\left[\left\{\sum_{j=1}^{m}\left(Y_{j}^{2}-1\right)\left(t_{j}-t_{j-1}\right)\right\}^{2}\right]$

$=E\left[\sum_{j=1}^{m} \sum_{k=1}^{m}\left(Y_{j}^{2}-1\right)\left(t_{j}-t_{j-1}\right)\left(Y_{k}^{2}-1\right)\left(t_{k}-t_{k-1}\right)\right]$

$=E\left[\sum_{j=k}\left(Y_{j}^{2}-1\right)^{2}\left(t_{j}-t_{j-1}\right)^{2}\right]+E\left[\sum_{j \neq k}\left(Y_{j}^{2}-1\right)\left(t_{j}-t_{j-1}\right)\left(Y_{k}^{2}-1\right)\left(t_{k}-t_{k-1}\right)\right]$

$=\sum_{j=1}^{\text {in }} E\left[\left(Y_{j}^{2}-1\right)^{2}\left(t_{j}-t_{j-1}\right)^{2}\right]$ because for each j, $E\left[\left(Y_{j}^{2}-1\right)\right]=0$.

Since the $Y_{j} s$ are independent and identically distributed,

$$
\begin{aligned}
& E\left[\left(S_{n}-(b-a)\right)^{2}\right]=E\left[\left(Y_{1}^{2}-1\right)^{2}\right] \sum_{j=1}^{m}\left(t_{j}-t_{j-1}\right)^{2} \\
& \leq E\left[\left(Y_{1}^{2}-1\right)^{2}\right](b-a)\left|\Pi_{n}\right| .
\end{aligned}
$$

Since $\left|\Pi_{n}\right| \rightarrow 0$ as $n \rightarrow \infty, E\left[\left(S_{n}-(b-a)\right)^{2}\right] \rightarrow 0$ as $n \rightarrow \infty$.

\section{Lemma 2.2.2}

Let $w(t)$ be a Brownian motion. Then for $0<t_{1}<t_{2}$

$$
\int_{t_{1}}^{t_{2}} w(t) d w(t)=\frac{1}{2}\left[\left(w\left(t_{2}\right)\right)^{2}-\left(w\left(t_{1}\right)\right)^{2}-\left(t_{2}-t_{1}\right)\right]
$$

or stated as a differential

$$
d(w(t))^{2}=d t+2 w(t) d w(t)
$$


Proof:

Let $\left[t_{1}, t_{2}\right]$ be partitioned with $\Pi_{n}=\left\{t_{1}=t_{1, n}<t_{2, n}<\cdots<t_{n, n}=t_{2}\right\}$. Then,

$$
\begin{aligned}
& \int_{t_{1}}^{t_{2}} w(t) d w(t)=\lim _{n \rightarrow \infty} \sum_{k=1}^{n-1} w\left(t_{k, n}\right)\left(w\left(t_{k+1, n}\right)-w\left(t_{k, n}\right)\right) \\
& =\frac{1}{2} \lim _{n \rightarrow \infty} \sum_{k=1}^{n-1}\left\{\left[\left(w\left(t_{k+1, n}\right)\right)^{2}-\left(w\left(t_{k, n}\right)\right)^{2}\right]-\left[w\left(t_{k+1, n}\right)-w\left(t_{k, n}\right)\right]^{2}\right\} \\
& =\frac{1}{2}\left(w\left(t_{2}\right)\right)^{2}-\frac{1}{2}\left(w\left(t_{1}\right)\right)^{2}-\frac{1}{2} \lim _{n \rightarrow \infty} \sum_{k=1}^{n-1}\left(w\left(t_{k+1, n}\right)-w\left(t_{k, n}\right)\right)^{2} .
\end{aligned}
$$

By Lemma 2.2.1, the limit above is $t_{2}-t_{1}$.

\section{Lemma 2.2.3}

Let $w(t)$ be a Brownian motion. Then $\int_{t_{1}}^{t_{2}} w(t) d t+\int_{t_{1}}^{t_{2}} t d w(t)=t_{2} w\left(t_{2}\right)-t_{1} w\left(t_{1}\right)$ or stated as a differential, $d(t w(t))=w(t) d t+t d w(t)$

Proof:

Let $\left\{t_{k, n}\right\}$ be a partition of $\left[t_{1}, t_{2}\right]$. Then

$\int_{t_{1}}^{t_{2}} w(t) d t=\lim _{n \rightarrow \infty} \sum_{k=1}^{n-1} w\left(t_{k+1, n}\right)\left(t_{k+1, n}-t_{k, n}\right)$ in probability

$\int_{t_{1}}^{t_{2}} t d w(t)=\lim _{n \rightarrow \infty} \sum_{k=1}^{n-1} t_{k, n}\left[w\left(t_{k+1, n}\right)-w\left(t_{k, n}\right)\right]$

Add (2.2.1) and (2.2.2)

$\int_{i_{1}}^{t_{2}} w(t) d t+\int_{i_{1}}^{t_{2}} t d w(t)=\lim _{n \rightarrow \infty} \sum_{k=1}^{n-1}\left[t_{k+1, n} w\left(t_{k+1, n}\right)-t_{k, n} w\left(t_{k, n}\right)\right]$ 


$$
=t_{2} w\left(t_{2}\right)-t_{1} w\left(t_{1}\right)
$$

The next two lemmas will be stated without proof. These are needed in the proof of the stochastic product rule. See Friedman [1] for proofs.

\section{Lemma 2.2.4:}

Let $f$ and $f_{n}$ be in $L^{2}[a, b]$ and suppose that

$$
\int_{a}^{b}\left|f_{n}(t)-f(t)\right|^{2} d t \stackrel{p}{\rightarrow} 0 \text { as } n \rightarrow \infty
$$

Then

$$
\int_{a}^{b} f_{n}(t) d w(t) \stackrel{P}{\rightarrow} \int_{a}^{b} f(t) d w(t)
$$

Lemma 2.2.5:

Let $f$ and $f_{n}$ be in $L^{2}[0, T]$ and assume that $\int_{0}^{T} f_{n}(t)-\left.f(t)\right|^{2} d t \stackrel{p}{\rightarrow} 0$ as $n \rightarrow \infty$. Then

$$
\sup _{0 \leq t \leq T}\left|\int_{0}^{t} f_{n}(s) d w(s)-\int_{0}^{t} f(s) d w(s)\right| \stackrel{P}{\rightarrow} 0 \text { as } n \rightarrow \infty .
$$

We now have all the facts needed to establish the stochastic product rule. 


\section{Lemma 2.2.6: Stochastic Product Rule}

Let $d \xi_{1}(t)=a_{1}(t) d t+b_{1}(t) d w(t)$

and $d \xi_{2}(t)=a_{2}(t) d t+b_{2}(t) d w(t)$ on $[0, T]$.

Then $d\left(\xi_{1}(t) \xi_{2}(t)\right)=\xi_{1}(t) d \xi_{2}(t)+\xi_{2}(t) d \xi_{1}(t)+b_{1}(t) b_{2}(t) d t$

Proof:

First, let $a_{i}$ and $b_{i}$ be constant for $i=1,2$. The result follows from Lemma 2.2.2 and

Lemma 2.2.3. This is easily extended to step functions, $a_{i}$ and $b_{i}$, because they are constant functions on sub-intervals.

In the general case, approximate $a_{i}$ and $b_{i}$ by non-anticipative step functions $a_{i, n}$ and $b_{i, n}$ so that

$$
\begin{aligned}
& \int_{0}^{T}\left|a_{i, n}(t)-a_{i}(t)\right| d t \rightarrow 0 \text { a.s., } \\
& \int_{0}^{T} b_{i, n}(t)-\left.b_{i}(t)\right|^{2} d t \rightarrow 0 \text { a.s. }
\end{aligned}
$$

Let $\xi_{i, n}(t)=\xi_{i}(0)+\int_{0}^{t} a_{i, n}(s) d s+\int_{0}^{t} b_{i, n}(s) d w(s)$. Then, by Lemma 2.2.5,

$$
\sup _{0 \leq t \leq T}\left|\xi_{i, n}(t)-\xi_{i}(t)\right| \stackrel{P}{\rightarrow} 0 \text { as } n \rightarrow \infty \text {. }
$$

Thus, there is a subsequence, $\xi_{i, n^{\prime}}$ such that

$$
\int_{t_{1}}^{t_{2}} \xi_{i, n^{\prime}}(t) b_{j, n^{\prime}}(t) d w(t) \stackrel{P}{\rightarrow} \int_{t_{1}}^{t_{2}} \xi_{i}(t) b_{j}(t) d w(t) \text { as } n^{\prime} \rightarrow \infty
$$

We also have: 
$\int_{t_{1}}^{t_{2}} \xi_{i, n}(t) a_{j, n}(t) d t \rightarrow \int_{t_{1}}^{t_{2}} \xi_{i}(t) a_{j}(t) d t$ a.s. and

$\int_{t_{1}}^{t_{2}} b_{1, n}(t) b_{2, n}(t) d t \rightarrow \int_{t_{1}}^{t_{2}} b_{1}(t) b_{2}(t) d t$ a.s.

The lemma follows from the above three equations.

We now have all the preliminary theory needed to prove Ito's Formula.

Theorem 2.2.7: Ito's Formula

Let $d \xi(t)=a(t) d t+b(t) d w(t)$ and let $f(x, t): \mathbb{R} \times[0, \infty] \rightarrow \mathbb{R}$ be a continuous function with continuous derivatives $f_{t}, f_{x}$, and $f_{x x}$. Then,

$$
\begin{aligned}
d f(\xi(t), t) & =\left[f_{t}(\xi(t), t)+f_{x}(\xi(t), t) a(t)+\frac{1}{2} f_{x x}(\xi(t), t) b^{2}(t)\right] d t \\
& +f_{x}(\xi(t), t) b(t) d w(t)
\end{aligned}
$$

Proof:

Step 1: For any integer $m \geq 2$,

$$
d(w(t))^{m}=m(w(t))^{m-1} d w(t)+\frac{1}{2} m(m-1)(w(t))^{m-2} d t
$$

Proof by induction:

$$
\text { case } m=2 \text { : by Theorem } 6, d(w(t))^{2}=2 w(t) d w(t)+d t \text {. }
$$


Suppose the statement is true for some $m \geq 2$. Then,

$$
\begin{aligned}
d(w(t))^{m+1} & =w(t) d(w(t))^{m}+(w(t))^{m} d w(t)+m(w(t))^{m-1} d t \\
& =m(w(t))^{m} d w(t)+\frac{1}{2} m(m-1)(w(t))^{m-1} d t \\
& +(w(t))^{m} d w(t)+m(w(t))^{m-1} d t \\
& =(m+1)(w(t))^{m} d w(t)+\frac{1}{2}(m+1) m(w(t))^{m-1} d t .
\end{aligned}
$$

Now, let $Q(x)$ be any polynomial. By the linearity property of the stochastic integral,

$d Q(w(t))=Q^{\prime}(w(t)) d w(t)+\frac{1}{2} Q^{\prime \prime}(w(t)) d t$

Step 2: Let $G(x, t)=Q(x) g(t)$ where $Q(x)$ is a polynomial and $g(t)$ is a continuously differentiable function for $t \geq 0$. By Lemma 2.2.6 and (2.2.3),

$$
\begin{aligned}
d G(w(t), t) & =Q(w(t)) d g(t)+g(t) d Q(w(t)) \\
& =\left[Q(w(t)) g^{\prime}(t)+\frac{1}{2} g(t) Q^{\prime \prime}(w(t))\right] d t \\
& +g(t) Q^{\prime}(w(t)) d w(t)
\end{aligned}
$$

or, stated as an integral

$$
\begin{aligned}
G\left(w\left(t_{2}\right)-t_{2}\right)-G\left(w\left(t_{1}\right), t_{1}\right)= & \int_{t_{1}}^{t_{2}}\left[G_{t}(w(t), t)+\frac{1}{2} G_{x x}(w(t), t)\right] d t \\
& +\int_{t_{1}}^{t_{2}} G_{x}(w(t), t) d w(t) .
\end{aligned}
$$

Step 3: Equation (2.2.4) holds for $G(x, t)=\sum_{i=1}^{m} f_{i}(x) g_{i}(t)$ where $f_{i}$ are polynomials and $g_{i}$ are continuously differentiable functions.

Now let $G_{n}(x, t)$ be polynomials in $x, t$ such that 


$$
\begin{aligned}
& \lim _{n \rightarrow \infty} G_{n}(x, t)=f(x, t) \\
& \lim _{n \rightarrow \infty} \frac{\partial}{\partial x} G_{n}(x, t)=f_{x}(x, t) \\
& \lim _{n \rightarrow \infty} \frac{\partial^{2}}{\partial x^{2}} G_{n}(x, t)=f_{x x}(x, t) \\
& \lim _{n \rightarrow \infty} \frac{\partial}{\partial t} G_{n}(x, t)=f_{l}(x, t)
\end{aligned}
$$

uniformly on a compact subset of $\mathbb{R} \times[0, \infty]$.

Thus,

$$
\begin{aligned}
& \int_{t_{1}}^{t_{2}}\left[\frac{\partial}{\partial t} G_{n}(w(t), t)+\frac{1}{2} \frac{\partial^{2}}{\partial x^{2}} G_{n}(w(t), t)\right] d t \\
& \rightarrow \int_{t_{1}}^{t_{2}}\left[f_{t}(w(t), t)+\frac{1}{2} f_{x x}(w(t), t)\right] d t \text { a. s. and } \\
& \lim _{n \rightarrow \infty} \int_{t_{1}}^{t_{2}}\left|\frac{\partial}{\partial x} G_{n}(w(t), t)-f_{x}(w(t), t)\right|^{2} d t=0 \text { a.s. }
\end{aligned}
$$

Taking the limit as $n \rightarrow \infty$,

$$
\begin{aligned}
f\left(w\left(t_{2}\right), t_{2}\right)-f\left(w\left(t_{1}\right), t_{1}\right)= & \int_{t_{1}}^{t_{2}}\left[f_{1}(w(t), t)+\frac{1}{2} f_{x}(w(t), t)\right] d t \\
& +\int_{t_{1}}^{t_{2}} f_{x}(w(t), t) d w(t) .
\end{aligned}
$$

Step 4: Equation (2.2.5) extends to the process $\Phi(w(t), t)=f\left(\xi_{1}+a_{1} t+b_{1} w(t), t\right)$ where $\xi_{1}, a_{1}, b_{1}$ are random variables measurable with respect to $\mathcal{F}_{t}$. In other words,

$$
\begin{aligned}
\Phi\left(w\left(t_{2}\right), t_{2}\right)-\Phi\left(w\left(t_{1}\right), t_{1}\right)= & \int_{t_{1}}^{t_{2}}\left[f_{t}(\tilde{\xi}(t), t)+a_{1} f_{x}(\tilde{\xi}(t), t)+\frac{1}{2} b_{1}^{2} f_{x x}(\tilde{\xi}(t), t)\right] d t \\
& +\int_{t_{1}}^{t_{2}} b_{1} f_{x}(\tilde{\xi}(t), t) d w(t)
\end{aligned}
$$

where $\tilde{\xi}(t)=\xi_{1}+a_{1} t+b_{1} w(t)$. 
Step 5: If $a(t), b(t)$ are step functions, then

$$
\begin{aligned}
f\left(\xi\left(t_{2}\right), t_{2}\right)-f\left(\xi\left(t_{1}, t_{1}\right)\right)= & \int_{t_{1}}^{t_{2}}\left[f_{t}(\xi(t), t)+a(t) f_{x}(\xi(t), t)+\frac{1}{2} b_{1}^{2}(t) f_{x x}(\xi(t), t)\right] d t \\
& +\int_{t_{1}}^{t_{2}} b(t) f_{x}(\xi(t), t) d w(t) .
\end{aligned}
$$

Denote successive intervals in $\left[t_{1}, t_{2}\right]$ by $I_{1}, I_{2}, \ldots, I_{k}$. Sum the integral in Step 4 over all the $I_{j} s$ and the above follows.

Step 6: Let $a_{i}, b_{i}$ be non-anticipative step functions such that

$$
\begin{aligned}
& \int_{0}^{T}\left|a_{i}(t)-a(t)\right| d t \rightarrow 0 \text { a.s. } \\
& \int_{0}^{T}\left|b_{i}(t)-b(t)\right|^{2} d t \stackrel{P}{\rightarrow} 0
\end{aligned}
$$

and let

$\xi_{i}(t)=\xi(0)+\int_{0}^{t} a_{i}(s) d s+\int_{0}^{t} b_{i}(s) d w(s)$

Then, $\sup _{0 \leq t \leq t}\left|\xi_{i}(t)-\xi(t)\right| \stackrel{P}{\rightarrow} 0$. Hence, there is a subsequence $\left\{i^{\prime}\right\}$ such that $\sup _{0 \leq \leq \leq T}\left|\xi_{i}(t)-\xi(t)\right| \rightarrow 0$ a. s. as $i=i^{\prime} \rightarrow \infty$. This and (2.2.7) imply that $\int_{0}^{T}\left|b_{i}(t) f_{x}\left(\xi_{i}(t), t\right)-b(t) f_{x}(\xi(t), t)\right|^{2} d t \stackrel{P}{\rightarrow} 0$ as $i=i^{\prime} \rightarrow \infty$.

It follows that 


$$
\int_{i_{1}}^{t_{2}} b_{i}(t) f_{x}\left(\xi_{i}(t), t\right) d w(t) \stackrel{P}{\rightarrow} \int_{t_{1}}^{t_{2}} b(t) f_{x}(\xi(t), t) d w(t) \text { as } i=i^{\prime} \rightarrow \infty
$$

From $(2.2 .6),(2.2 .7)$, and (2.2.8):

$$
\begin{aligned}
& \int_{t_{1}}^{t_{2}}\left[f_{i}\left(\xi_{i}(t), t\right)+a_{i}(t) f_{x}\left(\xi_{i}(t), t\right)+\frac{1}{2} b_{i}^{2}(t) f_{x x}\left(\xi_{i}(t), t\right)\right] d t \\
& \stackrel{P}{\rightarrow} \int_{t_{1}}^{t_{2}}\left[f_{t}(\xi(t), t)+a(t) f_{x}(\xi(t), t)+\frac{1}{2} b^{2}(t) f_{x x}(\xi(t), t)\right] d t \\
& \text { as } i=i^{\prime} \rightarrow \infty .
\end{aligned}
$$

Writing the integral at the end of Step 5 with $a=a_{i}, b=b_{i}, \xi=\xi_{i}$ and taking limits as $i=i^{t} \rightarrow \infty$, the formula in Step 5 follows for general $a$ and $b$.

\subsection{Girsanov's Theorem}

In many of the financial applications presented in this thesis, we will need to find a probability measure such that a particular stochastic process becomes a martingale. A martingale is defined as a stochastic process that has the property that the expected value is equal to the last observed value. That is, if a process $S(t)$ has been observed up to a time $t_{0}$, then for all $t>t_{0}, E[S(t)]=S\left(t_{0}\right)$. If a process is not a martingale with respect to a particular probability measure, it is sometimes possible to find another measure such that it is a martingale. The theorem that provides the means of making this transformation is Girsanov's Theorem. 
Let $h(t)$ be a random adapted path with $\int_{0}^{1}\left(h^{\prime}(s)\right)^{2} d s<\infty$ a. s. and let $w$ be Brownian motion with respect to the probability measure $\gamma$. Define $G(w)=\exp \left\{\int_{0}^{1} h^{\prime}(s) d w_{s}-\frac{1}{2} \int_{0}^{1}\left(h^{\prime}(s)\right)^{2} d s\right\}$ and assume that $E[G(w)]=1$. Let $\tilde{\gamma}$ be a probability measure such that $d \tilde{\gamma}=G(w) d \gamma$. Then, $w-h(w)$ has the same distribution with respect to $\tilde{\gamma}$ as $w$ has with respect to $\gamma$.

Proof:

Let $\gamma_{n}$ be a measure on $\mathbb{R}^{n}$ absolutely continuous with respect to Lebesgue measure, with Radon-Nikodym derivative $\frac{d \gamma_{n}}{d x}=f(x)$

Let $\phi: \mathbb{R}^{n} \rightarrow \mathbb{R}$ be a bounded continuous function such that $\int_{\mathbb{R}^{n}} \phi d \gamma_{n}=\int_{\mathbb{R}^{n}} \phi(x) f(x) d x$

Let $x=T(y)$ where $T: \mathbb{R}^{n} \rightarrow \mathbb{R}^{n}, T(y)=y-h(y)$ and $h_{k}(y)=h\left(y_{1}, \ldots, y_{k-1}\right)$. Then, $\int_{\mathbb{R}^{n}} \phi(x) f(x) d x=\int_{\mathbb{R}^{n}} \phi(T(y)) f(T(y)) J_{T}(y) d y$.

It can be demonstrated that $J_{r} \equiv 1$. Thus,

$$
\int_{\mathcal{F}^{n}} \phi d \gamma_{n}=\int_{\hat{f}^{n}} \phi(T(y)) \frac{f(T(y))}{f(y)} f(y) d y .
$$


Now, let the interval $[0,1]$ be partitioned by $\Pi_{m}=\left\{0=t_{0}<t_{1}<\cdots<t_{m}=1\right\}$ and

$$
\begin{aligned}
& f(y)=\exp \left\{-\sum_{i=1}^{m} \frac{\left(y_{i}-y_{i-1}\right)^{2}}{2\left(t_{i}-t_{i-1}\right)}\right\} \text {. Then, } \\
& \int_{\mathbb{R}^{n}} \phi d \gamma_{n}=\int_{\mathbb{R}^{n}} \phi(T(y)) \exp \sum_{i=1}^{m}\left\{\frac{\left(y_{i}-y_{i-1}\right)\left(h_{i}-h_{i-1}\right)}{t_{i}-t_{i-1}}-\frac{\left(h_{i}-h_{i-1}\right)^{2}}{2\left(t_{i}-t_{i-1}\right)}\right\} d \gamma_{n} .
\end{aligned}
$$

Therefore, the theorem is true for finite dimensional processes.

Define: $P_{m}: C_{0} \rightarrow \mathbb{R}^{n}$ by $P_{m}(w)=\left(w\left(t_{1}\right), \ldots, w\left(t_{m}\right)\right)$

and $Q_{m}: \mathbb{R}^{n} \rightarrow C_{0}$ by $Q_{m}\left(x_{1}, \ldots, x_{m}\right)=$ a piecewise linear path through $x_{1}, \ldots, x_{m}$.

Then, $Q_{m}\left(P_{m}(w)\right) \rightarrow w$ as $m \rightarrow \infty$ where $w$ is the Brownian motion.

Define: $h^{n}: \mathbb{R}^{n} \rightarrow \mathbb{R}^{n}$ by $h_{j}^{n}\left(P_{n}(w)\right) \equiv E\left[h\left(t_{j}\right) \mid w\left(t_{1}\right), \ldots, w\left(t_{n}\right)\right]$

As $n \rightarrow \infty$,

$$
\begin{aligned}
& h_{j}^{n} \rightarrow h\left(t_{j}\right) \\
& h^{n}\left(P_{n}(w)\right) \rightarrow h \\
& Q_{n}\left(h^{n}\left(P_{n}(w)\right)\right) \rightarrow Q_{n}\left(P_{n}(h(w))\right) \rightarrow h(w) .
\end{aligned}
$$

$\int \phi\left(Q_{n}\left(P_{n}(w)-h^{n} P_{n}(w)\right)\right) \times \exp \left\{\begin{array}{l}\frac{\left[\left(P_{n}(w)\right)_{i}-\left(P_{n}(w)\right)_{i-1}\right]}{t_{i}-t_{i-1}}\left[h_{i}^{n}\left(P_{n}(w)\right)-h_{i-1}^{n}\left(P_{n}(w)\right)\right] \\ -\frac{\left[h_{i}^{n}(P(w))-h_{i-1}^{n}(P(w))\right]^{2}}{2\left(t_{i}-t_{i-1}\right)}\end{array}\right\} d \gamma$

$=\int_{\mathbb{R}^{n}} \phi\left(Q_{n}(y)\right) d \gamma_{n}(y)$ by equation (2.3.1) 


$$
\begin{aligned}
& =\int_{C_{0}} \phi\left(Q_{n}\left(P_{n}(w)\right)\right) d \gamma(w) \\
& \rightarrow \int_{C_{0}} \phi(w) d \gamma(w) \text { as } n \rightarrow \infty
\end{aligned}
$$

One application of Girsanov's Theorem is that given a stochastic process that is Brownian motion plus drift with respect to a probability measure defined by $d \gamma$, we can find a probability measure defined by $d \tilde{\gamma}$ such that the stochastic process is Brownian motion with respect to the new measure. We will make use of this application in modeling financial derivatives.

\subsection{Stratonovich Calculus}

Stratonovich calculus is an alternative form of stochastic calculus. Any process that can be represented by an Ito integral can also be represented by a Stratonovich integral. In this section, we will present the definition of the Stratanovich integral and a formula to convert stochastic integrals from Ito form to Stratonovich form.

Gard [8] provides a definition of the Statonovich integral as follows:

\section{Definition 5: Stratonovich Integral}

Let $\Pi_{n}=\left\{t_{1, n}=t_{1}, t_{2, n}, \ldots t_{n, n}=t_{2}\right\}$ be a sequence of partitions of the interval, $\left[t_{1}, t_{2}\right]$ with $\operatorname{mesh}\left|\Pi_{n}\right| \rightarrow 0$ as $n \rightarrow \infty$. 


$$
\int_{t_{1}}^{t_{2}} f(t) \circ d w(t)=\lim _{n \rightarrow \infty} \sum_{i=1}^{n} \frac{1}{2}\left(f\left(t_{i+1, n}\right)+f\left(t_{i, n}\right)\right)\left(w\left(t_{i+1, n}\right)-w\left(t_{i, n}\right)\right) .
$$

This is similar to the definition of the Ito integral but we use the average of $f(t)$ at both endpoints of the interval instead of the value at the left endpoint. An important property of the Stratonovich integral is that it follows the same chain rule as in Newtonian calculus. The next theorem shows a relationship between Ito and Stratonovich integrals which proves useful in solving stochastic differential equations.

\section{Theorem 2.4.1}

Let $f(t)=\int_{0}^{t} g(s) d w(s)+\int_{0}^{t} h(s) d s$. Then

$$
\int_{t_{1}}^{t_{2}} f(t) \circ d w(t)=\int_{i_{1}}^{t_{2}} f(t) d w(t)+\frac{1}{2} \int_{t_{1}}^{t_{2}} g(t) d t
$$

Proof:

See Gard [8].

\subsection{Existence and Uniqueness of Solutions of Stochastic Differential Equations}

The following theorem from Gard [8] gives the conditions for the existence and uniqueness of stochastic differential equations.

\section{Theorem 2.5.1}

Given the stochastic differential equation on the interval $[0, T]$ : 
$d X(t)=f(t, X(t)) d t+g(t, X(t)) d w(t)$.

Suppose the following conditions are satisfied:

- The functions $f(t, x)$ and $g(t, x)$ are measurable with respect to $t$ and $x$ for $t \in[0, T]$ and $x \in \mathbb{R}$.

- There exists a constant $K$ such that for all $t \in[0, T]$ and $x, y \in \mathbb{R}$

$$
\begin{aligned}
& \circ|f(t, x)-f(t, y)|+|g(t, x)-g(t, y)| \leq K|x-y| \\
& \circ|f(t, x)|^{2}+|g(t, x)|^{2} \leq K^{2}\left(1+|x|^{2}\right)
\end{aligned}
$$

- $X(0)$ is independent of $w(t)$ for $t>0$ and $E\left[(X(0))^{2}\right]<\infty$.

Then there is a solution, $X(t)$ of $(2.5 .1)$ on $[0, T]$ which is continuous with probability 1 and such that $\sup _{[0, T]} E\left[(X(t))^{2}\right]<\infty$. Furthermore, the solution is pathwise unique. In other words: If $X$ and $Y$ are two such solutions, then $P\left(\sup _{[0, T]}|X(t)-Y(t)|=0\right)=1$.

Proof:

See Gard [8]. 


\section{Financial Models}

\subsection{Assumptions}

Throughout this thesis, we will make certain assumptions about the financial markets. The assumptions are the same assumptions used to derive the Black-Scholes-Merton model for pricing derivatives. These assumptions are listed in Hull [4] as follows:

1. The stock price follows the process: $S(t)=S(0) e^{\mu t+\sigma w(t)}$.

2. It is possible to short sell securities and the full proceeds of the transaction are available for use. This transaction entails borrowing a security, then selling it and repaying the security at a later time. Short selling is equivalent to buying a negative amount of the security.

3. There are no transaction costs or taxes.

4. All securities are perfectly divisible.

5. When modeling options we will assume that there are no dividends during the life of the option.

6. Security trading is continuous.

7. The risk-free interest rate, $r$, is constant over time and the same for all maturities.

8. There are no opportunities for risk free arbitrage. This will be defined below. 


\section{Arbitrage}

Risk free arbitrage is a situation in which an investor will realize a profit with certainty with no initial investment. We will assume throughout this thesis that arbitrage is not possible. This assumption is essential to the development of option pricing models.

\section{Financial Derivatives}

Derivatives are securities that derive their value from some underlying asset. These include options and futures. Some examples are

- Call option on a stock. This is the right but not the obligation to buy a share of a particular stock on or before a particular date, called the expiration date, for a predetermined price, called the exercise price. There are two common varieties of calls

- American. These can be exercised at any time until the expiration date

- European. These can only be exercised on the expiration date

- Put option on a stock. This is the right but not the obligation to sell a share of a particular stock on or before the expiration date for a predetermined exercise price. There are also American and European versions of put options.

- Futures contract on an asset such as a share of stock. This is a contract to buy (or sell) a share of a particular stock at a predetermined date for a predetermined price. Note that this is not an optional purchase. Once both parties enter a futures contract, the buyer is required to pay the predetermined price and the seller is required to deliver the asset. 
We will develop models to determine a price for European call options using the assumptions listed in this section.

\subsection{Pricing a European Call Option - Risk Neutral Pricing Method}

In this section, we will develop the price for a European call option on a stock with price, $S(t)$, exercise price, $K$, expiration date, $T$, and initial stock price, $S(0)$. We will use an approach called "risk neutral" pricing as presented in Baxter and Rennie [2]. The basic strategy of this method is to find the expectation of the present value of the option using "risk neutral" probabilities. The probability measure used to take expectation is not the actual probability measure of the stock but rather the probability measure that would exist in a risk neutral world. It turns out that the risk neutral probability measure is the measure such that the present value of underlying asset's price process is a martingale. We will find this new probability measure by invoking Girsanov's Theorem.

To clarify the presentation of this method, we will first present an example using a very simplified model of the stock price process. This example is similar to examples presented in [3] and [4]. 


\section{Example 3.2.1}

Let the current market price of 1 share of a particular stock be $S(0)=\$ 100$. Suppose that the future stock prices will follow a stochastic process such that the value at the end of 3 months will be $S_{u}=\$ 112$ with probability .75 or $S_{d}=\$ 89$ with probability .25 .

Furthermore, suppose that the risk free interest rate will be a constant $5 \%$ annual rate for the next 3 months.

Suppose you wish to purchase a European call option with this stock as the underlying asset with an expiration date 3 months from now and an exercise price of $K=\$ 105$. That is, in 3 months, you will have the right to purchase a share of the stock for $\$ 105$. What should the buyer be willing to pay for this option? What price should the seller be willing to accept?

We will show that the buyer should be willing to pay no more than $\$ 3.68$ and the seller should be willing to accept no less than $\$ 3.68$ for the call. This is true because a higher price would result in an arbitrage opportunity for the seller whereas a lower price would result in an arbitrage opportunity for the buyer.

For example, suppose the call is sold for $\$ 3.00$. Then the buyer has an opportunity to make an arbitrage profit. He can purchase 1,000 calls, short sell 308 shares of the stock and invest the difference at the risk free interest rate. The cash flows resulting from this transaction are summarized in Table 3.2.1 below: 


\begin{tabular}{|lccc|}
\hline Table 3.2.1 & $\begin{array}{c}\text { Initial } \\
\text { Cash Flow }\end{array}$ & $\begin{array}{c}\text { Final Cash Flow } \\
\text { if stock goes up }\end{array}$ & $\begin{array}{c}\text { Final Cash Flow } \\
\text { if stock goes down }\end{array}$ \\
buy 1000 calls & $-3,000$ & 7,000 & 0 \\
short sell 308 stocks & 30,800 & $-34,496$ & $-27,500$ \\
invest at the risk free rate & $-27,800$ & 28,150 & 28,150 \\
TOTAL & 0 & 654 & 650 \\
\hline
\end{tabular}

So, the buyer makes a profit regardless of the final stock price and does not need to invest any of his own money. The proceeds from the short sale of the stocks will finance the purchase of the calls and the investment at the risk free interest rate.

On the other hand, suppose the call is sold for $\$ 4.00$. Then the seller has an arbitrage opportunity. He can sell 1,000 calls, purchase 308 shares of stock and borrow the difference at the risk free rate. The cash flows resulting from these transactions are summarized in Table 3.2.2.

\begin{tabular}{|lccc|}
\hline Table 3.2.2 & $\begin{array}{c}\text { Initial } \\
\text { Cash Flow }\end{array}$ & $\begin{array}{c}\text { Final Cash Flow } \\
\text { if stock qoes up }\end{array}$ & $\begin{array}{c}\text { Final Cash Flow } \\
\text { if stock goes down }\end{array}$ \\
short sell 1000 calls & 4,000 & $-7,000$ & 0 \\
buy 308 stocks & $-30,800$ & 34,496 & 27,500 \\
borrow at the risk free rate & 26,800 & $-27,137$ & $-27,137$ \\
TOTAL & 0 & 359 & 363 \\
\hline
\end{tabular}


The seller makes a risk free profit with no initial investment.

We could test various call prices until we find the unique arbitrage free price but there is a more straightforward method. We will find probabilities such that the present value of the stock price is a martingale and use them to calculate the present value of the expected cash flows.

If the present value of the stock price is a martingale

then $E\left[e^{-r T} S(T)\right]=e^{-r T}\left(p S_{u}+(1-p) S_{d}\right)=S(0)$. Thus, the risk neutral probability that the stock price increases is $p=\frac{e^{r T} S(0)-S_{d}}{S_{u}-S_{d}}$. Substituting the values from our example, $p=0.533$.

At time $\mathrm{T}$, if the final stock price is $S_{d}$, then the value of the call is 0 because $\$ 89$ is less than the strike price, $\$ 105$. If the final stock price is $S_{u}$ at time $\mathrm{T}$, then the call is worth $S_{u}-K=112-105=7$. The expected present value using the risk neutral probabilities is $C=e^{-(.25)(.05)}(.533)(7)=3.68$

This is the arbitrage free price of the call. Note that if we calculated the expected present value using the actual probability that the price goes up, we get $C=e^{-(.25)(.05)}(.75)(7)=5.18$. 
From Table 3.2.2, it is clear that this price would create an arbitrage opportunity for the seller of the call.

This method of pricing an option is known as the "risk neutral" method because it uses probabilities that would exist in a risk neutral world. In other words, if investors did not require additional return on their investment to compensate for risk, then the expected value of the final price would be the initial price plus risk free interest. In this makebelieve world, the probability that the final price is $\$ 112$ would be 0.533 instead of 0.75 .

In real markets, investors purchasing risky assets such as stocks demand a return in excess of the risk free rate to compensate them for the additional risk. In the context of pricing options, this excess retum is irrelevant hecause it can be eliminated by hedging strategies as shown in Tables 3.2.1 and 3.2.2.

The surprising fact established in this example is that the price of the call does not depend on the rate of return of the stock. Instead, it depends solely on the spread of the final stock prices and the risk free interest rate. In a more realistic model of the behavior of the stock price, the price of the call would depend only on the volatility of the stock price and the interest rate. 


\section{Section 3.3 Risk Neutral Pricing with Geometric Brownian Motion}

Let's proceed to a more realistic model of the stock price process: Geometric Brownian Motion.

Stock price process: $S(t)=S(0) e^{\left(\mu-\sigma^{2} / 2\right) t+\sigma w(t)}$. Expressed as a differential, this is

$d S=\mu S d t+\sigma S d w(t)$

Bond process: The price process for a risk free zero-coupon bond, such as a U. S.

Treasury bond, with a face value of $\$ 1.00$ can be represented as $B(t)=e^{r t}$.

We will be interested in the process for the present value of a stock. This is the ratio of the stock price to the value of a bond, $\tilde{S}(t)=S(t) / B(t)$. By Ito's Formula (Theorem 2.2.7),

$$
d \tilde{S}=(\mu-r) \tilde{S} d t+\sigma \tilde{S} d w(t)
$$

We want to find a probability measure such that $\tilde{S}(t)$ is a martingale. By Girsanov's Theorem, with $h^{\prime}(t)=-(\mu-r) / \sigma$, the process can be written with a new Brownian motion, $w^{B}$ such that 
$d \tilde{S}=(\mu-r) \tilde{S} d t+\sigma \tilde{S}\left(d w^{B}(t)-\frac{\mu-r}{\sigma} d t\right)$. This reduces to $d \tilde{S}=\sigma \tilde{S} d w^{B}(t)$ which is a

martingale. Using the new probability measure, the process for the stock price becomes

$S(t)=S(0) \exp \left\{\left(r-\frac{1}{2} \sigma^{2}\right) t+\sigma w^{D}(t)\right\}$

We can now take the expected value of the cash flows resulting from a call at maturity with respect to the new probability measure and calculate the call price as the present value of this expectation. The pdf corresponding to the risk neutral probability measure is $f(x)=\frac{e^{-\frac{1}{2} \frac{w^{2}}{T}}}{\sqrt{2 \pi T}}$, In other words, it is the pdf of a Gaussian random variable with mean 0 and variance $T$.

The value of the call at time 0 is

$$
\begin{aligned}
C(0) & =e^{-r T} E[\max \{S(T)-K, 0\}] \\
& =e^{-r T} \int_{-\infty}^{\infty} \max \left\{S(0) e^{\left(r-\frac{1}{2} \sigma^{2}\right) t+\sigma w}-K, 0\right\} \frac{e^{\frac{1}{2} \frac{w^{2}}{T}}}{\sqrt{2 \pi T}} d w .
\end{aligned}
$$

To evaluate the integral, we will define the following variables:

$$
\begin{gathered}
d=\frac{\ln (S(0) / K)+\left(r-\frac{\sigma^{2}}{2}\right) T}{\sigma \sqrt{T}} \\
y=-w / \sqrt{T}, d y=-d w / \sqrt{T} \\
u=-w / \sqrt{T}-\sigma \sqrt{T}, d u=-d w / \sqrt{T} .
\end{gathered}
$$


We now have:

$C(0)=S(0) \int_{-\infty}^{d+\sigma \sqrt{T}} \frac{e^{-\frac{u^{2}}{2}}}{\sqrt{2 \pi}} d u-K e^{-r T} \int_{-\infty}^{d} \frac{e^{-\frac{y^{2}}{2}}}{\sqrt{2 \pi}} d y$.

The solution is:

$$
C(0)=S(0) \Phi(d+\sigma \sqrt{T})-K e^{-r T} \Phi(d)
$$

where $\Phi(\cdot)$ is the standard normal cumulative distribution function.

In the next section, we will obtain the same result by solving a partial differential equation.

\subsection{Pricing a European Call Option - Partial Differential Equation Method - Assuming Geometric Brownian Motion}

The risk neutral method is simple to apply and appeals to financial intuition. However, it is difficult to apply if we assume models of the underlying stock price process other than geometric Brownian motion. Risk neutral pricing is also difficult to apply to more complex options such as American calls and puts. An American option can be exercised at any time until the expiration date, so its value does not depend only on the payoff at expiration, making the risk neutral valuation more complex. A more commonly used pricing method, the Black-Scholes partial differential equation method is more difficult to apply but can be adapted to alternative stock models and more complex options. 
The derivation and solution of the Black-Scholes PDE presented in this section is based on Wilmott, Howison and Dewynne [3]. We will refer to the simple binomial example in section 3.2 and illustrated in tables 3.2 .1 and 3.2.2. In this example we see that, given the simple binomial probability distribution, both the buyer and the seller of a call can hedge their risk against both outcomes of the final stock price by constructing a risk free portfolio. In this section, we will construct a risk free portfolio from the buyer's point of view, but this time we will assume that the stock price follows geometric Brownian motion:

$d S=\mu S d t+\sigma S d w(t)$

We will denote the value of the risk free portfolio by $\Pi$. The buyer will purchase a call option and short sell some unknown amount of shares, denoted by $\Delta$, needed to hedge the risk of the underlying stock process. The value of the portfolio is given by $\Pi(S, t)=C(S, t)-\Delta S(t)$

The return on the portfolio over an infinitesimal time step is $d \Pi(t)=d C(t)-\Delta d S(t)$. Applying Ito's Formula with (3.4.1) and (3.4.2): $d \Pi=\sigma S\left(\frac{\partial C}{\partial S}-\Delta\right) d w(t)+\left(\mu S \frac{\partial C}{\partial S}+\frac{1}{2} \sigma^{2} S^{2} \frac{\partial^{2} C}{\partial S^{2}}+\frac{\partial C}{\partial t}-\mu \Delta S\right) d t$ 
If we choose $\Delta=\frac{\partial C}{\partial S}$ at the beginning of each time step as the amount of stock to short sell, (3.4.3) reduces to $d \Pi=\left(\frac{\partial C}{\partial t}+\frac{1}{2} \sigma^{2} S^{2} \frac{\partial^{2} C}{\partial S^{2}}\right) d t$

The portfolio is entirely free of risk since the contribution of $d w(t)$ has been eliminated from the portfolio's return. Notice that this requires continuously rebalancing the composition of the portfolio. This is the reason we need to assume that security trading is continuous.

Since the portfolio is risk free, it will earn a risk free rate of retum, $r$, in an efficient financial market. Expressed mathematically,

$$
\begin{aligned}
& d \Pi=r \Pi d t \\
& \left(\frac{\partial C}{\partial t}+\frac{1}{2} \sigma^{2} S^{2} \frac{\partial^{2} C}{\partial S^{2}}\right) d t=r\left(C-\frac{\partial C}{\partial S}\right) d t .
\end{aligned}
$$

The above reduces to the Black-Scholes PDE:

$$
\frac{\partial C}{\partial t}+\frac{1}{2} \sigma^{2} S^{2} \frac{\partial^{2} C}{\partial S^{2}}+r S \frac{\partial C}{\partial S}-r C=0
$$

The boundary values corresponding to a European call option with excercise price $K$ and expiration $T$ are:

$$
C(S, T)=\max (S-K, 0) \text { the final condition }
$$


$C(0, t)=0$

$C(S, t)-S$ as $S \rightarrow \infty$.

Now we have a boundary value problem that we will solve by the methodology presented in Wilmott, Howison and Dewynne [3]. First, we will make some changes of variables to transform the Black-Scholes equation from a backward diffusion equation into a forward diffusion equation which has a straightforward solution. The changes of variable also make the variables dimensionless.

$S=K e^{x}, t=T-\tau /\left(\frac{1}{2} \sigma^{2}\right), C=K \nu(x, \tau)$. These changes of variables result in

$\frac{\partial v}{\partial \tau}=\frac{\partial^{2} v}{\partial x^{2}}+(b-1) \frac{\partial v}{\partial x}-b v$ where $b=r / \frac{1}{2} \sigma^{2}$.

The direction of time has reversed so that the final condition has now become an initial condition as follows:

$$
\begin{aligned}
& C(S, T)=\max (S-K, 0) \\
& K v(x, 0)=C(S, T)=\max (S-K, 0)=\max \left(K e^{x}-K, 0\right) \\
& v(x, 0)=\max \left(e^{x}-1,0\right) .
\end{aligned}
$$

Next, we set $\nu=e^{\alpha_{x}+\beta \tau} u(x, \tau)$ which leads to

$$
\beta u+\frac{\partial u}{\partial \tau}=\alpha^{2} u+2 \alpha \frac{\partial u}{\partial x}+\frac{\partial^{2} u}{\partial x^{2}}+(b-1)\left(\alpha u+\frac{\partial u}{\partial x}\right)-b u
$$


We can simplify this by choosing $\alpha=-\frac{1}{2}(b-1), \beta=-\frac{1}{4}(b+1)^{2}$. The function $v$ becomes $v=e^{-\frac{1}{2}(b-1) x-\frac{1}{4}(b+1)^{2} \tau} u(x, \tau)$ and the equation simplifies to

$\frac{\partial u}{\partial \tau}=\frac{\partial^{2} u}{\partial x^{2}}$ for $-\infty<x<\infty, \tau>0$

with final condition

$u(x, 0)=u_{0}(x)=\max \left(e^{\frac{1}{2}(b+1) x}-e^{\frac{1}{2}(b-1) x}, 0\right)$

This is the heat equation for an infinite bar with diffusivity equal to unity. This problem has been studied for many years. The following solution is presented in Powers [5].

We assume that $u(x, \tau)=X(x) T(\tau) \cdot(3.4 .10)$ becomes

$$
\frac{X^{\prime \prime}(x)}{X(x)}=\frac{T^{\prime}(\tau)}{T(\tau)}=-\lambda^{2}
$$

This can be separated into two ordinary differential equations:

$X^{\prime \prime}(x)+\lambda^{2} X(x)=0$

which has solution $X(x)=A(\lambda) \cos (\lambda x)+B(\lambda) \sin (\lambda x)$

and $T^{\prime}(\tau)+\lambda^{2} T(\tau)=0$

which has solution $T(\tau)=e^{-\lambda^{2} \tau}$.

Multiplying the solutions to (3.4.12) and (3.4.13), the solution to (3.4.10) is 
$u(x, \tau)=[A(\lambda) \cos (\lambda x)+B(\lambda) \sin (\lambda x)] e^{-\lambda^{2} \tau}$.

Applying the initial condition,

$u_{0}(x)=\int_{0}^{\infty} A(\lambda) \cos (\lambda x)+B(\lambda) \sin (\lambda x) d \lambda$.

This is the Fourier integral representation of $u_{0}$. Thus,

$$
\begin{aligned}
& A(\lambda)=\frac{1}{\pi} \int_{-\infty}^{\infty} u_{0}(x) \cos (\lambda x) d x \\
& B(\lambda)=\frac{1}{\pi} \int_{-\infty}^{\infty} u_{0}(x) \sin (\lambda x) d x .
\end{aligned}
$$

Substituting the above expressions into (3.4.14),

$$
\begin{aligned}
& u(x, \tau)=\frac{1}{\pi} \int_{0}^{\infty}\left[\int_{-\infty}^{\infty} u_{0}(s) \cos (\lambda s) d s \cos (\lambda x)+\int_{-\infty}^{\infty} u_{0}(s) \sin (\lambda s) d s \sin (\lambda x)\right] \exp \left(-\lambda^{2} \tau\right) d \lambda \\
& u(x, \tau)=\frac{1}{\pi} \int_{0}^{\infty} \int_{-\infty}^{\infty} u_{0}(x)[\cos (\lambda s) \cos (\lambda x)+\sin (\lambda s) \sin (\lambda x)] d s \cdot \exp \left(-\lambda^{2} \tau\right) d \lambda \\
& u(x, \tau)=\frac{1}{\pi} \int_{-\infty}^{\infty} u_{0}(x)\left(\int_{0}^{\infty} \cos (\lambda(s-x)) \cdot \exp \left(-\lambda^{2} \tau\right) d \lambda\right) d s .
\end{aligned}
$$

The inner integral equals

$$
\sqrt{\frac{\pi}{4 \tau}} \exp \left[\frac{-(s-x)^{2}}{4 \tau}\right] \text {. }
$$

Thus, the final solution to the heat problem is

$$
u(x, \tau)=\frac{1}{2 \sqrt{\pi \tau}} \int_{-\infty}^{\infty} u_{0}(s) \exp \left[\frac{-(s-x) 2}{4 \tau}\right] d s
$$

Next, we need to transform the problem back to the original call pricing problem. Define $z=(s-x) / \sqrt{2 \tau}$. The above integral reduces to 


$$
\begin{aligned}
& u(x, \tau)=\frac{1}{2 \pi} \int_{-\infty}^{\infty} u_{0}(z \sqrt{2 \tau}+x) e^{-\frac{1}{2} z^{2}} d z \\
& =\frac{1}{\sqrt{2 \pi}} \int_{-x / \sqrt{2 \tau}}^{\infty} \exp \left\{\frac{1}{2}(b+1)(x+z \sqrt{2 \tau})\right\} \exp \left\{-\frac{1}{2} z^{2}\right\} d z \\
& -\frac{1}{\sqrt{2 \pi}} \int_{-x / \sqrt{2 \tau}}^{\infty} \exp \left\{\frac{1}{2}(b-1)(x+z, \sqrt{2 \tau})\right\} \exp \left\{-\frac{1}{2} z^{2}\right\} d z \\
& =\frac{\exp \left\{\frac{1}{2}(b+1) x+\frac{1}{4}(k+1)^{2} \tau\right\}}{\sqrt{2 \pi}} \int_{-x / \sqrt{2 \tau}-\frac{1}{2}(b+1) \sqrt{2 \tau}}^{\infty} \exp \left\{\frac{1}{2} p^{2}\right\} d p \\
& -\frac{\exp \left\{\frac{1}{2}(b-1) x+\frac{1}{4}(k+1)^{2} \tau\right\}}{\sqrt{2 \pi}} \int_{-x / \sqrt{2 \tau}-\frac{1}{2}(b-1) \sqrt{2 \tau}}^{\infty} \exp \left\{\frac{1}{2} p^{2}\right\} d p \\
& =\exp \left(\frac{1}{2}(b+1) x+\frac{1}{4}(b+1)^{2} \tau\right) \Phi\left(\frac{x}{\sqrt{2 \tau}}+\frac{1}{2}(b+1) \sqrt{2 \tau}\right) \\
& -\exp \left(\frac{1}{2}(b-1) x+\frac{1}{4}(b-1)^{2} \tau\right) \Phi\left(\frac{x}{\sqrt{2 \tau}}+\frac{1}{2}(b-1) \sqrt{2 \tau}\right) .
\end{aligned}
$$

Substituting our original variables

$$
\begin{aligned}
& x=\ln (S / K), \tau=\frac{1}{2} \sigma^{2}(T-t), C=K \nu(x, \tau), \\
& v(x, \tau)=e^{-\frac{1}{2}(b-1) x-\frac{1}{4}(b+1)^{2} \tau} u(x, \tau)
\end{aligned}
$$

we obtain the same solution as in Section 3.3. 


\section{Alternative Stock Models}

The model of the stock price process represented by equation (3.3.1) is a commonly used model but it is does not fit all stock price processes. Financial analysts must consider other models in order to price derivative assets accurately. In this section, we will present two alternative stock models. The first model is presented in a 1975 paper by Cox and Ross [6] and is discussed in Hull [4]. There is a solution for the price of a call via a partial differential equation but no solution via risk neutral valuation.

\subsection{The Cox and Ross Model}

In a paper written in 1975 , Cox and Ross [7] present an alternative model of the stock process. The model is based on the assumption that the change in stock price is the sum of a deterministic drift term proportional to the stock price and a jump diffusion process. The jump diffusion process represents packets of information arriving at random times and having a random effect on the stock price. This is very close to what happens in real markets: financial analysts receive new information about a company at random points in time and incorporate the new information into their valuations of the company.

The stock process is modeled by the stochastic differential equation $d s=\mu S d t+(k-1) \lambda S d t+(1-\lambda d t) 0=\mu S d t+(k-1) \lambda S d t$ 
The stock price increases with a deterministic drift rate $\mu$ and changes by an amount $(k-1) S$ with probability $\lambda d t$ or a change of 0 with probability $1-\lambda d t$. If we let $\mathrm{k}$ be a random variable with distribution $k= \begin{cases}k^{+}>1 & \text { with probability } \pi^{+} \\ k^{-}<1 & \text { with probability } \pi^{*}\end{cases}$

In the limit, as $k^{+} \rightarrow 1, k^{-\cdots} \rightarrow 1$, and $\lambda \rightarrow \infty$, the model becomes:

$d S=\mu S d t+\sigma \sqrt{S} d w(t)$

Assuming this model for the stock price process, we can solve for the price of a call by deriving a partial differential equation, then solving it, similar to the procedure used in section 3.4 .

First, we form a risk free portfolio by purchasing a call option with value $C(S, t)$ and short selling $\Delta$ shares of stock. The value of the portfolio is $\Pi(S, t)=C(S, t)-\Delta S(t)$

Next, we apply Ito's Formula to calculate the instantaneous return on the portfolio $d \Pi=\sigma \sqrt{S}\left(\frac{\partial C}{\partial S}-\Delta\right) d w(t)+\left(\mu S \frac{\partial C}{\partial S}+\frac{1}{2} \sigma^{2} S \frac{\partial^{2} C}{\partial S^{2}}+\frac{\partial C}{\partial t}-\mu \Delta S\right) d t$

Choose $\Delta=\frac{\partial C}{\partial S}$ so that the retum is free of risk, $d \Pi=\left(\mu S \frac{\partial C}{\partial S}+\frac{1}{2} \sigma^{2} S \frac{\partial^{2} C}{\partial S^{2}}+\frac{\partial C}{\partial t}-\mu \Delta S\right) d t$ 
The assumption of no risk free arbitrage implies that the above portfolio will earn the risk free rate of return. This leads to the equation

$$
\left(\mu S \frac{\partial C}{\partial S}+\frac{1}{2} \sigma^{2} S \frac{\partial^{2} C}{\partial S^{2}}+\frac{\partial C}{\partial t}-\mu \Delta S\right) d t=r \Pi d t=r\left(C-\frac{\partial C}{\partial S} S\right) d t
$$

This equation reduces to

$$
\frac{\partial C}{\partial t}+\frac{1}{2} \sigma^{2} S \frac{\partial^{2} C}{\partial S^{2}}+r S \frac{\partial C}{\partial S}-r C=0
$$

Note that this is almost the same as the original Black-Scholes equation except that the $\sigma^{2}$ term has a factor of $S$ instead of $S^{2}$. The final value and boundary conditions are also the same as the original Black-Scholes problem. However, the solution is much more difficult. Cox and Ross [7] provide the following solution:

$$
C(S, t)=S \sum_{n=0}^{\infty} \frac{(n+1) e^{-y} y^{n} G(n+2, \theta K)}{\Gamma(n+2)}-K e^{-r(T-t)} \sum_{n=0}^{\infty} \frac{e^{-y} y^{n+1} G(n+1, \theta K)}{\Gamma(n+2)}
$$

where

$$
\begin{aligned}
& \theta=\frac{2 r}{\sigma^{2}\left[e^{r(T-t)-1}\right]}, \\
& y=\theta S e^{r(T-t)}, \text { and } \\
& G(m, x)=[\Gamma(m)]^{-1} \int_{x}^{\infty} e^{-z} z^{m n-1} d z .
\end{aligned}
$$




\subsection{Stock Model Using a Stratonovich Differential}

In this section, we will present a model developed by Dr. Denis Bell of the University of North Florida. The model is similar to the Cox and Ross model but the stochastic differential is a Statonovich differential instead of an Ito differential. The advantage is that if a Stratonovich differential is assumed, then the stochastic differential equation is solvable and we can apply the risk neutral valuation method to value a call option.

Henceforth, the model will be referred to as the "fractional" model. The fractional model is derived using the following theorem.

\section{Theorem 4.2.1}

Let $\mathrm{r}$ be the risk free interest rate and let the price process of a stock be represented by an Ito process:

$$
d S=a(t, S) d t+b(t, S) d w(t)
$$

Then there exists a probability measure $\tilde{P}$ with respect to which $S^{*}(t)=e^{-r t} S(t)$ is a martingale. Let $\tilde{E}$ denote the expectation with respect to $\tilde{P}$. Then for any functional $F$ defined on the space of paths, $\tilde{E}[F(S)]=E[F(\tilde{S})]$ where $\tilde{S}$ satisfies the stochastic differential equation $d \tilde{S}=r \tilde{S} d t+b(t, \tilde{S}) d w(t)$ 
Proof:

Using Ito's Formula, we have

$$
\begin{aligned}
& d S^{*}=-r e^{-r t} S d t+e^{-r t} d S \\
& =-r e^{-r t} S d t+e^{-r t}(a d t+b d w) \\
& =e^{-r t} b\left\{\left(\frac{a-r S}{b}\right) d t+d w\right\} .
\end{aligned}
$$

Set $d \tilde{w}=\left(\frac{a-r S}{b}\right) d t+d w$. Then, by Girsanov's Theorem, there exists a probability

measure $\tilde{P}$ with respect to which $\tilde{w}$ is a Brownian motion. This implies that $S^{*}(t)$ is a martingale. Substituting

$$
\begin{aligned}
& d w=d \tilde{w}-\left(\frac{a-r S}{b} d t\right) \text { into }(4.2 .1), \text { we have } \\
& d S=r S d t+b(t, S) d \tilde{w}(t)
\end{aligned}
$$

Thus the distribution of $S$ with respect to $\tilde{P}$ is the same as the distribution of $\tilde{S}$ with respect to $P$.

To develop our alternative model of the stock, we start with the stochastic differential equation

$$
d \tilde{S}=r S d t+\sigma \tilde{S}^{p} \circ d w(t)
$$

where the stock process is given by $S(t)=G(\tilde{S}(t), t)$. Note that the differential in (4.2.2) is a Stratonovich differential. 
Ito's Formula yields

$$
\begin{aligned}
& d S(t)=\frac{\partial G}{\partial t}+\frac{\partial G}{\partial S} d \tilde{S}(t)+\frac{1}{2} \frac{\partial^{2} G}{\partial S^{2}}(d \tilde{S}(t))^{2} \\
& =\left[\frac{\partial G}{\partial t}+\left(r \tilde{S}+\frac{p \sigma^{2}}{2} \tilde{S}^{2 p-1}\right) \frac{\partial G}{\partial S}+\frac{\sigma^{2} \tilde{S}^{2 p}}{2} \frac{\partial^{2} G}{\partial S^{2}}-r G\right] d t+\frac{\partial G}{\partial S} \sigma \tilde{S}^{p} d w(t)
\end{aligned}
$$

This implies that

$$
d S^{*}(t)=e^{-r t}\left[\frac{\partial G}{\partial t}+\left(r \tilde{S}+\frac{p \sigma^{2}}{2} \tilde{S}^{2 p-1}\right) \frac{\partial G}{\partial S}+\frac{\sigma^{2} \tilde{S}^{2 p}}{2} \frac{\partial^{2} G}{\partial S^{2}}-r G\right] d t+e^{-r t} \frac{\partial G}{\partial S} \sigma \tilde{S}^{p} d w(t)
$$

Recall that the condition of no risk free arbitrage implies that $S^{*}(t)$ is a martingale. This condition will be satisfied if we choose $\mathrm{G}$ to satisfy the partial differential equation:

$\frac{\partial G}{\partial t}+\left(r s+\frac{p \sigma^{2} S^{2 p-1}}{2}\right) \frac{\partial G}{\partial S}+\frac{\sigma^{2} s^{2 p}}{2} \frac{\partial^{2} G}{\partial S^{2}}=r G$

We must also impose the condition: $G(\tilde{S}(0), 0)=S(0)$.

We consider the special case, $p=1 / 2:(4.2 .3)$ becomes

$$
\frac{\partial G}{\partial t}+\left(r s+\frac{\sigma^{2}}{4}\right) \frac{\partial G}{\partial S}+\frac{\sigma^{2} S}{2} \frac{\partial^{2} G}{\partial S^{2}}=r G
$$

The solution is

$$
\begin{aligned}
& G(S)=S+\frac{\sigma^{2}}{4 r} \text { which yields } \\
& S(t)=\tilde{S}(t)+\frac{\sigma^{2}}{4 r} \text { and } S^{*}(t)=\tilde{S}^{*}(t)+\frac{\sigma^{2}}{4 r}
\end{aligned}
$$


Equation (4.2.5) provides a transformation from $\tilde{S}$ to $S$. Solving the stochastic differential equation (4.2.2), we have

$$
\tilde{S}(t)=e^{r t}\left\{\frac{\sigma}{2} \int_{b}^{r} e^{-r s / 2} d w(s)+\left(S-\frac{\sigma^{2}}{4 r}\right)^{1 / 2}\right\}^{2} .
$$

Equations (4.2.6) and (4.2.5) yield

$$
S^{*}(t)=\tilde{S}^{*}(t)+\frac{\sigma^{2}}{4 r}=X^{2}+\frac{\sigma^{2}}{4 r} .
$$

where $X(t)=\sqrt{\tilde{S}^{*}(t)} \cdot X(t)$ is a Normal random variable with mean $e^{-\frac{n t}{2}}\left(S(0)-\frac{\sigma^{2}}{4 r}\right)^{1 / 2}$ and variance $\frac{\sigma^{2}}{4 r}\left(e^{r t}-1\right)$

The Ito equation corresponding to (4.2.5) is

$$
d S=r S d t+\sigma \sqrt{S-\frac{\sigma^{2}}{4 r}} d w(t)
$$

Equation (4.2.8) is the Ito equation of the stock process after applying Theorem 4.2.1. The model of the actual stock price is

$$
d S=\mu S d t+\sqrt{S-\frac{\sigma^{2}}{4 r}} d w(t)
$$

Now, we can use the risk neutral valuation to solve for the value of a European call option. As usual, let the option have exercise price $K$ and expiration date $T$. We have $C(t)=e^{-r t} E[\max (S(t)-K, 0)]$. 
$E[\max (S(t)-K, 0)]=\int_{-\infty}^{\infty} \max \left(x^{2}+\frac{\sigma^{2}}{4 r}-K, 0\right) \frac{1}{\sqrt{2 \pi \nu}} \exp \left\{-\frac{(x-m)^{2}}{2 v^{2}}\right\} d x$

where $m=e^{r / 2}\left(S(0)-\frac{\sigma^{2}}{4 r}\right)^{1 / 2}$ and $v^{2}=\frac{\sigma^{2}}{4 r}\left(e^{r t}-1\right)$. Let $z=\frac{x-m}{v}, d_{1}=\frac{-\sqrt{K-\frac{\sigma^{2}}{4 r}}-m}{v}$,

and $d_{2}=\frac{\sqrt{K-\frac{\sigma^{2}}{4 r}}-m}{v}$

Then,

$$
\begin{aligned}
& E[\max (S(t)-K, 0)]=\int_{-\infty}^{d_{1}}\left(v^{2} z^{2}+2 m v z+m^{2}+\frac{\sigma^{2}}{4 r}-K\right) \frac{1}{\sqrt{2 \pi}} \exp \left(-\frac{z^{2}}{2}\right) d z+ \\
& \int_{d_{2}}^{\infty}\left(v^{2} z^{2}+2 m v z+m^{2}+\frac{\sigma^{2}}{4 r}-K\right) \frac{1}{\sqrt{2 \pi}} \exp \left(-\frac{z^{2}}{2}\right) d z \\
& =v^{2} \int_{-\infty}^{d_{1}} z^{2} \frac{1}{\sqrt{2 \pi}} \exp \left(-\frac{z^{2}}{2}\right) d z+2 m v \int_{-\infty}^{d_{1}} z \frac{1}{\sqrt{2 \pi}} \exp \left(-\frac{z^{2}}{2}\right) d z+\left(m^{2}+\frac{\sigma^{2}}{4 r}-K\right) \int_{-\infty}^{d_{1}} \frac{1}{\sqrt{2 \pi}} \exp \left(-\frac{z^{2}}{2}\right) d z \\
& +v^{2} \int_{d_{2}}^{\infty} z^{2} \frac{1}{\sqrt{2 \pi}} \exp \left(-\frac{z^{2}}{2}\right) d z+2 m v \int_{d_{2}}^{\infty} z \frac{1}{\sqrt{2 \pi}} \exp \left(-\frac{z^{2}}{2}\right) d z+\left(m^{2}+\frac{\sigma^{2}}{4 r}-K\right) \int_{d_{2}}^{\infty} \frac{1}{\sqrt{2 \pi}} \exp \left(-\frac{z^{2}}{2}\right) d z \\
& C(t)=e^{-r t}\left\{\frac{\left(v^{2} d_{2}+2 m \nu\right) e^{-d_{2} / 2}-\left(v^{2} d_{1}+2 m v\right) e^{-d_{1} / 2}}{\sqrt{2 \pi}}+\left(m^{2}+\frac{\sigma^{2}}{4 r}-k+v^{2}\right)\left[1-\Phi\left(d_{2}\right)+\Phi\left(d_{1}\right)\right]\right\} .
\end{aligned}
$$




\section{Fitting Data to Stock Models}

In this section, we will use actual stock data to estimate the parameters for the geometric Brownian motion model and the fractional model. Next, we will use the estimated parameters to calculate values of European call options. The results of these calculations are displayed in Table 5.1 at the end of this section.

The data used were the end-of-day adjusted closing prices for a sample of the Standard and Poor 100 stocks. Each sample in Table 5.1 represents a 50 trading day period for one stock. The stock prices were adjusted to remove the distorting effects of dividends and stock splits. We used the estimated volatility of the stock during one 50 day period as a predictor of the volatility for the subsequent 50 days. The estimated volatility was used to price a European call with an expiration date 50 days hence.

\section{Fitting Data to the Geometric Brownian Motion Model}

To estimate the parameters $\mu$ and $\sigma$ for the geometric Brownian motion model, we follow the methodology presented by Hull [4]. Recall that the solution to (3.3.1) is $S(t)=S(0) e^{\left(\mu-\sigma^{2} / 2\right) t+\sigma w(t)}$. This gives the price of a stock at time $t$. Let time be measured in trading days. We have the following expression for the logarithm of the daily change in the price of the stock.

$u_{k}=\log _{a}(S(k) / S(k-1))=\left(\mu-\sigma^{2} / 2\right)+\sigma[w(k)-w(k-1)]$. 
By the properties of Brownian motion, each $u_{k}$ is an independent normal random variable with mean $\mu-\sigma^{2} / 2$ and variance $\sigma^{2}$. We used the procedures available in the SAS programming language to fit values to the mean and variance of the distribution of $u_{k}$ using the method of maximum likelihood.

The null hypothesis is:

$H_{0}: u_{k} \sim N(\mu, \sigma)$.

The null hypothesis was tested using the Shapiro-Wilk statistic at the $95 \%$ confidence level. Note that $\mu$ and $\sigma$ estimated with these data represent the daily mean and volatility.

\section{Fitting Data to the Fractional Model}

We used the same stock data to test the fractional model presented in section 4.2. Due to the complexity of the model, the parameters were estimated using numerical methods. As in the case of the geometric Brownian motion model, we calculated Normal random variables that are functions of the stock prices and the assumed parameters. Recall the stochastic differential equation for the model, equation 4.2 .2 with $\mathrm{p}=1 / 2$.

$$
d \tilde{S}=r \tilde{S} d t+\sigma \tilde{S}^{1 / 2} \circ d w(t)
$$

The Ito equation corresponding to 5.2 .1 is

$$
d S=\mu S d t+\sigma \sqrt{S-\frac{\sigma^{2}}{4 r}} d w(t)
$$

Since the data are discrete, we use a discrete approximation to 5.2.2: 
$S_{k+1}-S_{k}=\mu S_{k}+\sigma \sqrt{S_{k}-\frac{\sigma^{2}}{4 r}}\left(w_{k+1}-w_{k}\right)$.

where $S_{k}$ and $w_{k}$ are the stock price and the value of the Brownian motion on the $\mathrm{k}^{\text {th }}$ day.

From 5.2.3, we can calculate standard normal random variables for each trading day:

$u_{k}=\frac{S_{k+1}-S_{k}-\mu S_{k}}{\sigma \sqrt{S_{k}-\frac{\sigma^{2}}{4 r}}}$.

In order to estimate the parameters $\mu$ and $\sigma$, we must use numerical methods to solve the system of equations:

$\frac{1}{N} \sum_{k=1}^{N} u_{k}=0$

$\frac{1}{N-1} \sum_{k=1}^{N} u_{k}^{2}=1$

Here $N$ is the number of available values of $u_{k}$. We must also select a reasonable estimate of the risk-free interest rate since $u_{k}$ is a function of $r$. In this case, we selected $3.0 \%$ annually as the risk-free interest rate.

Using Newton's Method in Mathematica, we attempted to solve (5.2.4) and (5.2.5) for the same data used in section 5.1. The solution to the equations does not always exist but we recorded the results in cases where Newton's Method converged. Next, we calculated values of $u_{k}$ based on the selected interest rate and the estimated parameters. ShapiroWilk statistics were computed in order to test whether the $u_{k} s$ have a normal distribution. 
The null hypothesis is:

$H_{0}: u_{k} \sim N(\mu, \sigma)$

The results of the tests are shown in table 5.1 along with the results for the geometric Brownian motion model. 


\begin{tabular}{|c|c|c|c|c|c|c|c|c|}
\hline & $\begin{array}{l}\text { San } \\
\text { Rest }\end{array}$ & $\begin{array}{l}\text { nple } \\
\text { ults of } T\end{array}$ & $\begin{array}{l}\text { tand } \\
\text { for No }\end{array}$ & $\begin{array}{l}\text { le } 5.1 \\
\text { and } \\
\text { lity and }\end{array}$ & $\begin{array}{l}\text { or } 1 \\
\text { urope }\end{array}$ & $\begin{array}{l}100 \mathrm{St} \\
\text { an Call }\end{array}$ & $\begin{array}{l}\text { cks } \\
\text { ices }\end{array}$ & \\
\hline & $\dot{c}$ & $\hat{j}$ & Reje & $\mathrm{H}_{0}$ ? & Initial & Call & Europear & Call Price \\
\hline $\begin{array}{l}\text { Sample } \\
\text { Number }\end{array}$ & $\begin{array}{c}\text { Standard } \\
\text { Model }\end{array}$ & $\begin{array}{c}\text { Fractional } \\
\text { Model }\end{array}$ & $\begin{array}{c}\text { Standard } \\
\text { Model }\end{array}$ & $\begin{array}{c}\text { Fractional } \\
\text { Model }\end{array}$ & $\begin{array}{l}\text { Stock } \\
\text { Price }\end{array}$ & $\begin{array}{c}\text { Exercise } \\
\text { Price }\end{array}$ & $\begin{array}{c}\text { Standard } \\
\text { Model }\end{array}$ & $\begin{array}{c}\text { Fractional } \\
\text { Model }\end{array}$ \\
\hline 1 & 0.00887 & 0.05569 & $N$ & $N$ & 29.50 & 30.00 & 0.59 & 0.60 \\
\hline 2 & 0.00859 & 0.06359 & $N$ & $N$ & 37.43 & 38.00 & 0.75 & 0.80 \\
\hline 3 & 0.00995 & 0.10320 & $N$ & $N$ & 62.88 & 64.00 & 1.43 & 1.51 \\
\hline 4 & 0.01500 & 0.10320 & $Y$ & $Y$ & 66.37 & 68.00 & 2.26 & 1.38 \\
\hline 5 & 0.00805 & 0.06429 & $Y$ & $Y$ & 50.41 & 51.00 & 1.01 & 1.03 \\
\hline 6 & 0.00989 & 0.09488 & $N$ & $N$ & 50.63 & 52.00 & 0.96 & 1.04 \\
\hline 7 & 0.00841 & 0.06879 & $N$ & $N$ & 50.17 & 51.00 & 0.95 & 0.98 \\
\hline 8 & 0.00970 & 0.08700 & $\mathrm{~N}$ & $\mathrm{~N}$ & 46.76 & 48.00 & 0.87 & 0.94 \\
\hline 9 & 0.00771 & 0.06585 & $N$ & $N$ & 56.91 & 58.00 & 0.91 & 0.95 \\
\hline 10 & 0.00867 & 0.07537 & $\mathrm{~N}$ & $\mathrm{~N}$ & 55.71 & 57.00 & 0.95 & 0.98 \\
\hline 11 & 0.01518 & 0.07537 & $Y$ & Y & 57.10 & 58.00 & 2.19 & 1.17 \\
\hline 12 & 0.00859 & 0.05095 & $N$ & $N$ & 26.68 & 27.00 & 0.57 & 0.58 \\
\hline 13 & 0.00776 & 0.06325 & $Y$ & $Y$ & 49.86 & 51.00 & 0.73 & 0.78 \\
\hline 14 & 0.00931 & 0.08078 & $\mathrm{~N}$ & $\mathrm{~N}$ & 54.35 & 55.00 & 1.27 & 1.29 \\
\hline 15 & 0.00873 & 0.07959 & $\mathrm{~N}$ & $\mathrm{~N}$ & 55.10 & 56.00 & 1.10 & 1.18 \\
\hline 16 & 0.00761 & 0.06745 & $N$ & $N$ & 63.61 & 65.00 & 0.93 & 0.96 \\
\hline 17 & 0.00972 & 0.07174 & $N$ & $N$ & 31.35 & 32.00 & 0.65 & 0.71 \\
\hline 18 & 0.00942 & 0.06786 & $N$ & $N$ & 30.71 & 31.00 & 0.76 & 0.82 \\
\hline 19 & 0.00774 & 0.04689 & $\mathrm{~N}$ & $\mathrm{~N}$ & 33.66 & 34.00 & 0.67 & 0.64 \\
\hline 20 & 0.00567 & 0.03588 & $\mathrm{~N}$ & $\mathrm{~N}$ & 35.30 & 36.00 & 0.36 & 0.37 \\
\hline 21 & 0.00930 & 0.10796 & $Y$ & $Y$ & 90.48 & 92.00 & 1.93 & 2.02 \\
\hline 22 & 0.00914 & 0.07253 & $Y$ & $Y$ & 44.09 & 45.00 & 0.85 & 0.88 \\
\hline 23 & 0.00708 & 0.04846 & $N$ & $N$ & 43.83 & 45.00 & 0.50 & 0.48 \\
\hline 24 & 0.00856 & 0.06525 & $\mathrm{~N}$ & $\mathrm{~N}$ & 39.64 & 40.00 & 0.90 & 0.96 \\
\hline 25 & 0.00905 & 0.07299 & $\mathrm{~N}$ & $\mathrm{~N}$ & 46.35 & 47.00 & 1.01 & 1.04 \\
\hline 26 & 0.00825 & 0.06538 & $\mathrm{~N}$ & $\mathrm{~N}$ & 47.04 & 48.00 & 0.80 & 0.83 \\
\hline 27 & 0.00966 & 0.11348 & $\mathrm{~N}$ & $\mathrm{~N}$ & 79.66 & 81.00 & 1.78 & 1.92 \\
\hline 28 & 0.00828 & 0.08899 & $\mathrm{~N}$ & $\mathrm{~N}$ & 86.94 & 89.00 & 1.37 & 1.43 \\
\hline 29 & 0.00836 & 0.09100 & $\mathrm{~N}$ & $\mathrm{~N}$ & 87.81 & 90.00 & 1.36 & 1.43 \\
\hline 30 & 0.00578 & 0.05883 & $N$ & $N$ & 94.45 & 96.00 & 1.10 & 1.11 \\
\hline 31 & 0.00840 & 0.05089 & $\mathrm{~N}$ & $\mathrm{~N}$ & 27.40 & 28.00 & 0.46 & 0.48 \\
\hline 32 & 0.00852 & 0.05283 & $N$ & $N$ & 28.34 & 29.00 & 0.47 & 0.49 \\
\hline 33 & 0.00678 & 0.03953 & $\mathrm{~N}$ & $\mathrm{~N}$ & 29.17 & 30.00 & 0.30 & 0.30 \\
\hline 34 & 0.00898 & 0.05580 & $N$ & $N$ & 24.73 & 25.00 & 0.57 & 0.61 \\
\hline 35 & 0.00952 & 0.09802 & $N$ & $N$ & 81.46 & 83.00 & 3.71 & 1.68 \\
\hline 36 & 0.00945 & 0.08464 & $N$ & $\mathrm{~N}$ & 50.57 & 52.00 & 0.87 & 0.94 \\
\hline 37 & 0.00737 & 0.06088 & $\mathrm{~N}$ & $\mathrm{~N}$ & 52.80 & 54.00 & 0.72 & 0.77 \\
\hline 38 & 0.00915 & 0.06676 & $\mathrm{~N}$ & $\mathrm{~N}$ & 35.04 & 36.00 & 0.59 & 0.63 \\
\hline 39 & 0.00875 & 0.06208 & $\mathrm{~N}$ & $\mathrm{~N}$ & 34.77 & 35.00 & 0.85 & 0.89 \\
\hline 40 & 0.00991 & 0.08059 & $Y$ & $Y$ & 37.49 & 38.00 & 0.91 & 0.97 \\
\hline
\end{tabular}




\section{Conclusions}

We have presented proofs of several important results from stochastic calculus and demonstrated their use in pricing a European call option. We have shown that the price of a call option does not depend on the rate of return of the underlying stock but rather on its volatility. This was true in the case of all three stock models presented in this thesis. Therefore, it is important to have a good estimate of the volatility of the price but it is not necessary to have an estimate of the stock's rate of return. The financial rationale for this conclusion is that the risk of the financial derivative can be hedged by forming a risk free portfolio. Knowing that the portfolio earns the risk free interest rate enables us to calculate the market price of the derivative.

In section 5, we used historical stock prices to estimate the volatilities of actual stocks. The Shapiro-Wilk statistics indicate that most of these stocks fit both the geometric Brownian motion model and the fractional model. The null hypothesis that the data were normally distributed was rejected in the same cases for each model. The estimated volatilities were used to calculate the prices of European call options. The fractional model generally produced higher prices for the call option. This indicates that the fractional model implies more risk for the seller of the call compared to the geometric Brownian motion model.

There are many opportunities for further research in mathematical finance. Possible topics include alternative models of the stock price process, pricing more complex 
options, for example American options, and varying any of the assumptions of the BlackScholes-Merton model listed in section 3. One could develop models that consider transaction costs and dividends. The assumption of a constant interest rate that is equal across maturities could be relaxed. Any variation of the original assumptions could lead to a new financial model that might have practical applications. Also, there is a need for research in reliable procedures for fitting volatilities to the stock models. For some of the stocks in our sample we were not able to obtain estimated volatilities for the fractional model because Newton's Method did not converge. It is possible that other numerical methods would have yielded estimated volatilities. 


\section{References}

[1] Avner Friedman, Stochastic Differential Equations and Applications, Academic Press, Inc., New York, 1975.

[2] Martin Baxter, Andrew Rennie, Financial Calculus - An Introduction to Derivative Pricing, Cambridge University Press, Cambridge, 1996.

[3] Paul Wilmott, Sam Howison, Jeff Dewynne, The Mathematics of Financial Derivatives, Press Syndicate of the University of Cambridge, New York, New York, 1996.

[4] John C. Hull, Options, Futures and Other Derivatives, 4th ed., Prentice-Hall, Upper Saddle River, NJ, 2000 ,

[5] Powers, David L., Boundary Value Problems, 2nd ed., Academic Press, Inc, New York, New York, 1979.

[6] John C. Cox and Stephen A. Ross, The Valuation of Options for Alternative Stock Processes, Journal of Financial Economics 3 (1976) 145-166, North-Holland Publishing Company.

[7] Denis Bell, Ph.D. Private communications

[8] Thomas C. Gard, Introduction to Stochastic Differential Equations, Marcel Dekker, Inc., New York, New York, 1988. 


\section{Vita}

Scott Stelljes was born in Fremont, Nebraska

He earned a Bachelor of

Science degree in Mathematics from the State University of New York in May, 1987. He served in the United States Air Force from 1981 to 1990 . He has worked in the insurance industry as a casualty actuary since 1990 . 